\title{
Head-to-Head Prospective Comparison of Quantitative Lung Scintigraphy and Segment Counting in Predicting Pulmonary Function in Lung Cancer Patients Undergoing Video-Assisted Thoracoscopic Lobectomy
}

\author{
Elite Arnon-Sheleg*1, Ori Haberfeld*2,3, Ran $\operatorname{Kremer}^{2,3}$, Zohar Keidar ${ }^{2,3}$, and Michal Weiler-Sagie ${ }^{2}$ \\ ${ }^{1}$ Galilee Medical Center, Nahariya, Israel; ${ }^{2}$ Rambam Medical Center, Haifa, Israel; and ${ }^{3}$ Rappaport Faculty of Medicine, Technion- \\ Israel Institute of Technology, Haifa, Israel
}

Prediction of postoperative pulmonary function in lung cancer patients before tumor resection is essential for patient selection for surgery and is conventionally done with a nonimaging segment counting method (SC) or 2-dimensional planar lung perfusion scintigraphy (PS). The purpose of this study was to compare quantitative analysis of PS to SPECT/CT and to estimate the accuracy of SC, PS, and SPECT/CT in predicting postoperative pulmonary function in patients undergoing lobectomy. Methods: Seventy-five non-small cell lung cancer patients planned for lobectomy were prospectively enrolled $(68 \%$ male; average age, $68.1 \pm$ $8 \mathrm{y})$. All patients completed tests of preoperative forced expiratory volume capacity in $1 \mathrm{~s}$ (FEV1) and diffusing capacity of the lungs for carbon monoxide (DLCO), as well as $99 \mathrm{mTc}$-macroaggregated albumin PS and SPECT/CT quantification. A subgroup of 60 patients underwent video-assisted thoracoscopic lobectomy and measurement of postoperative FEV1 and DLCO. Relative uptake of the lung lobes estimated by PS and SPECT/CT was compared. Predicted postoperative FEV1 and DLCO were derived from SC, PS, and SPECT/CT. Prediction results were compared between the different methods and the true postoperative measurements in patients who underwent lobectomy. Results: Relative uptake measurements differed significantly between PS and SPECT/CT in right lung lobes, with a mean difference of $-8.2 \pm 3.8,18.0 \pm 5.0$, and $-11.5 \pm 6.1$ for right upper, middle, and lower lobes, respectively $(P<0.001)$. The differences between the methods in the left lung lobes were minor, with a mean difference of $-0.4 \pm 4.4(P>0.05)$ and $-2.0 \pm 4.0(P<0.001)$ for left upper and lower lobes, respectively. No significant difference and a strong correlation $(R=0.6-0.76$, $P<0.001$ ) were found between predicted postoperative lung function values according to SC, PS, SPECT/CT, and the actual postoperative FEV1 and DLCO. Conclusion: Although lobar quantification parameters differed significantly between PS and SPECT/CT, no significant differences were found between the predicted postoperative lung function results derived from these methods and the actual postoperative results. The additional time and effort of SPECT/CT quantification may not have an added value in patient selection for surgery. SPECT/CT may be advantageous in patients planned for right lobectomy, but further research is warranted.

Received Jul. 31, 2019; revision accepted Nov. 6, 2019.

For correspondence or reprints contact: Elite Arnon-Sheleg, Galilee Medical Center, P.O. Box 21, Nahariya, 22100, Israel.

E-mail: elite.arnon@gmail.com

${ }^{*}$ Contributed equally to this work.

Published online Dec. 20, 2019.

COPYRIGHT (C 2020 by the Society of Nuclear Medicine and Molecular Imaging.
Key Words: lung function; perfusion; SPECT/CT; VATS lobectomy; lung cancer

J Nucl Med 2020; 61:981-989

DOI: 10.2967/jnumed.119.234526

\section{$\mathbf{L}$}

ung cancer is the most common cause of death among oncologic patients worldwide (1). Resection is the treatment of choice for early-stage non-small cell lung cancer (2). Because of a common risk factor (smoking), many lung cancer patients have coexisting lung disease resulting in reduced pulmonary function and an increased risk of perioperative morbidity and mortality (3).

Estimation of respiratory reserve is an essential element of preoperative evaluation. Candidates for lung resection undergo respiratory evaluation by pulmonary function testing with measurement of FEV1 (forced expiratory volume in $1 \mathrm{~s}$ ) and DLCO (diffusing capacity of the lungs for carbon monoxide) $(3,4)$. Patients with an FEV1 or DLCO that is less than $80 \%$ of the predicted value are defined as high-risk patients and require that pulmonary function be estimated after the operation by calculating the predicted postoperative FEV1 and DLCO (ppoFEV1 and ppoDLCO) $(5,6)$. A predicted value of less than $30 \%$ is considered a significant risk for perioperative mortality or morbidity, and thus, the operation is contraindicated (5). The predicted postoperative lung function can be calculated simply by multiplying the preoperative FEV1 or DLCO by the fractional number of lung segments that will remain after the operation (7). This method, called segment counting (SC), is considered reliable, but some studies have found that it is inaccurate because it ignores the possibility that some of the segments have reduced function and it tends to underestimate the ppoFEV1 $(7,8)$.

Radionuclide perfusion lung scanning with ${ }^{99 \mathrm{~m}} \mathrm{Tc}$-labeled macroaggregated albumin can be used to estimate and quantify the regional distribution of lung function. The contribution of a lobe or an entire lung can be determined by drawing regions of interest on planar perfusion images. Typically, the data from quantitative radionuclide perfusion scans are reported as the percentage of function contributed by the 6 lung regions: upper third, middle third, and lower third of each hemithorax. These data, combined with the preoperative lung function value and the location and planned extent of surgical resection, permit a calculation of the 
ppoFEV1/DLCO value. The ppoFEV1 derived from planar perfusion scintigraphy (PS) has shown a fair correlation with the spirometry-measured postoperative FEV1 in pneumonectomy patients (9); however, less is known about using perfusion scintigraphy to estimate postlobectomy lung function. SPECT can provide tomographic images of lung perfusion. The comparison of SPECT (without concomitant CT) and PS has shown no significant advantage in postoperative lung function prediction (10-12), possibly because of attenuation effects and the inability to trace the lobar anatomic boundaries since the lung fissures are not visible in the study. In recent years, several studies have attempted to compare the postoperative prediction of lung function between PS and SPECT/CT perfusion scans with various methodologies (13-19). Most of these studies had small numbers of lobectomy patients, and some did not compare the predicted results with the actual postoperative lung function tests. Some of the studies found SPECT/CT-based calculations to be superior, but some showed no difference from planar imaging.

This study aimed to compare lung function quantification with PS and SPECT/CT and to estimate the accuracy of SC, PS, and SPECT/CT for the prediction of postoperative lung function, in a selected and homogeneous group of early-stage non-small cell lung cancer patients undergoing video-assisted thoracoscopic lobectomy (VATS).

\section{MATERIALS AND METHODS}

Patients who had been admitted for VATS lobectomy or bilobectomy because of non-small cell lung cancer with reduced pulmonary function (FEV1 or DLCO $<80 \%$ of predicted) were included in the study. Seventy-five patients were prospectively enrolled from December 2016 to April 2018 (mean age \pm SD, $68.1 \pm 8$ y; 51 men [68\%]; 66 smokers [88\%]). The study was approved by the Institutional Ethics Committee, and each subject signed an informed consent form.
All included patients underwent preoperative spirometry (for FEV1) and DLCO 1-14 d before surgery (pre-FEV1 and pre-DLCO) and showed reduced lung function (FEV1 or DLCO $<80 \%$ of predicted). Lung perfusion scanning was performed $1-5 \mathrm{~d}$ before surgery after a slow intravenous injection of $150 \mathrm{MBq}$ of ${ }^{99 \mathrm{~m} T c-m a c r o a g g r e g a t e d ~ a l-~}$ bumin with the patient supine. Imaging data was acquired on a dualhead $\gamma$-camera (Discovery 670; GE Healthcare) using a low-energy high-resolution parallel-hole collimator and an energy window of 140 $\mathrm{keV} \pm 10 \%$ for emission counts. PS consisted of a static posterior and anterior acquisition of 500,000 counts on a $256 \times 256$ matrix with a square pixel size of $1.74 \mathrm{~mm}$. SPECT was performed with projection data every $6^{\circ}$ for $360^{\circ}$ of rotation with a matrix of $128 \times 128$, a square pixel size of $4.45 \mathrm{~mm}$, and an acquisition duration of $15 \mathrm{~s}$ per projection. The SPECT acquisition was followed by a helical CT scan at a peak voltage of $120 \mathrm{kV}$ and adaptive tube current (50-180 mA using GE Healthcare Smart mA with a noise index of 24.6). The pitch was 1.375 , with $1 \mathrm{~s}$ per rotation. Image slice thickness was $3.75 \mathrm{~mm}$, and the matrix size was $512 \times 512$ for a 50 -cm-diameter transverse field of view. CT was reconstructed using filtered backprojection and a softtissue filter. SPECT was reconstructed with CT attenuation correction using ordered-subset expectation maximization with 2 iterations, 10 subsets, Butterworth postfiltering (order, 10; cutoff frequency, 0.48 $\mathrm{cm}^{-1}$ ), and an isotropic voxel size of $4.45 \mathrm{~mm}$. PS was processed for quantitative perfusion analysis using automatic software provided by the Xeleris workstation (GE Healthcare). The software divides both lungs into 3 regions of interest and calculates the relative uptake in each region (zone) using the geometric mean of the anterior and posterior images (Fig. 1A). In the right lung, the upper, middle, and lower zones correspond to the right upper lobe (RUL), right middle lobe (RML), and right lower lobe (RLL), respectively. In the left lung, the upper zone and half of the middle zone correspond to the left upper lobe (LUL) and the lower zone, and half of the middle zone corresponds to the left lower lobe (LLL). Quantitative analysis of the SPECT/CT image data was done with semiautomatic software, Qlung, provided by GE Healthcare (Fig. 1B). The software requires the reader to

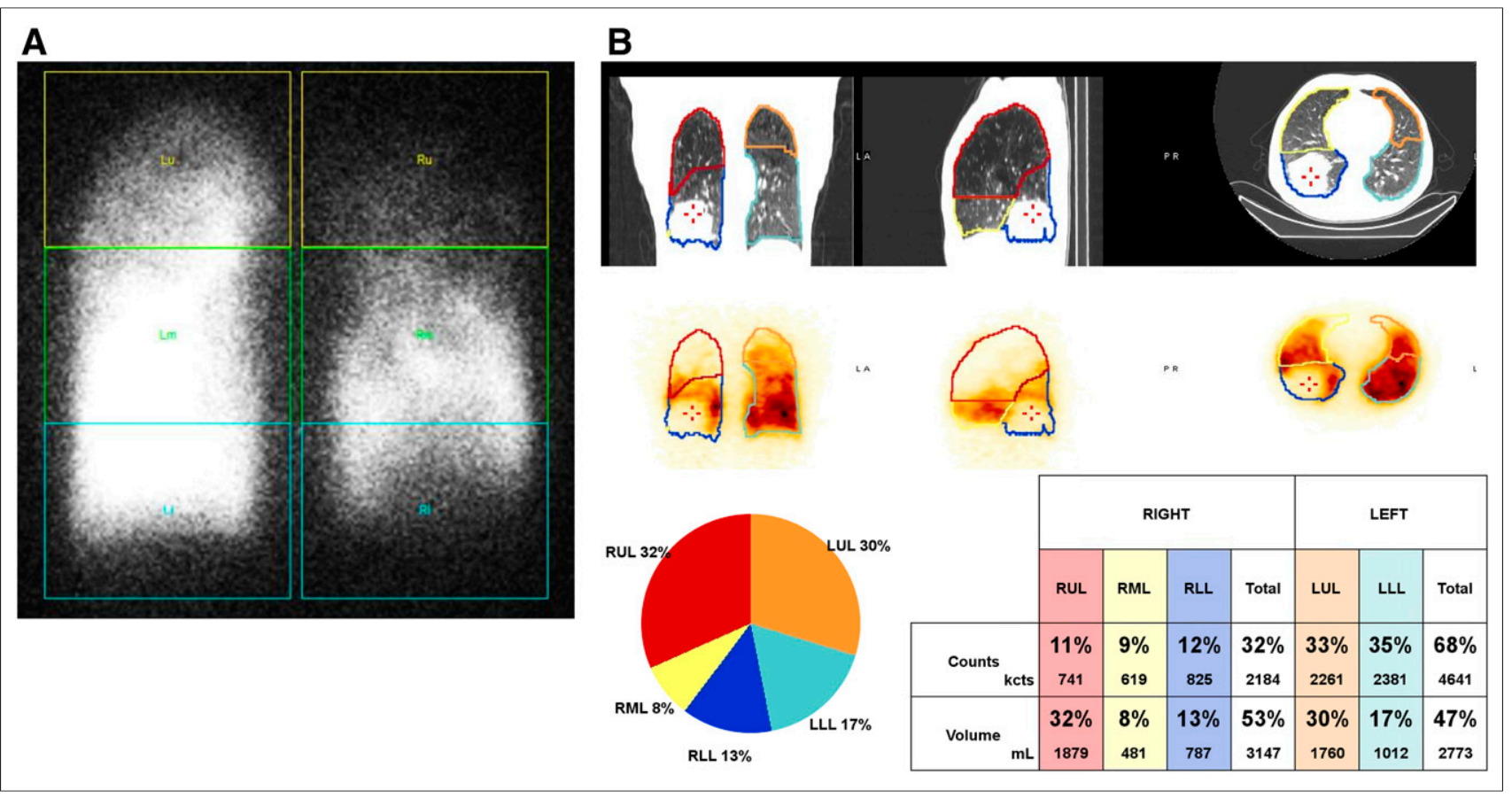

FIGURE 1. Methods of quantitative analysis of perfusion images in patient with RLL tumor. (A) Posterior view of planar study with automatic lung division into 6 zones. (B) Qlung quantitative analysis of SPECT/CT. 
mark each lung fissure in (at least) 2 places on the CT images. The software then automatically draws volumetric regions of interest for each lobe and calculates its relative uptake. The relative uptake was calculated for all 5 lobes in both methods (PS and SPECT/CT).

The predicted postoperative lung function values (ppoFEV1 and ppoDLCO) were calculated by 3 methods: SC, PS, and SPECT/CT. $\mathrm{SC}$ was calculated by multiplying the preoperative lung function tests by the predicted fraction of segments the patient will have after resection (8). The total number of segments in both lungs is 19 ( 3 in the RUL, 2 in the RML, 5 in the RLL, 5 in the LUL, and 4 in the LLL):

ppoFEV1 or ppoDLCO

$=\frac{\text { pre-FEV1 } \text { or pre-DLCO } \times(19-\text { number of excised segments })}{19}$.

PS was calculated by multiplying the preoperative lung function tests by the predicted residual functioning lung according to the planar quantification (20):

ppoFEV1 or ppoDLCO

$=\frac{\text { pre-FEV } 1 \text { or pre-DLCO } \times(100-\% \text { perfusion of planned resection })}{\text {. }}$.

SPECT/CT was calculated similarly to PS-predicted values using the percentage of perfused lung to be resected based on the SPECT/CT quantification. Using the predicted lung function results from SC and PS, patients with either a ppoFEV1 or a ppoDLCO of less than $30 \%$ were defined as being at very high risk and were excluded from surgical treatment.

Of the 75 enrolled patients, a subgroup of 60 underwent VATS lobectomy. Within a month after the operation, all patients in this subgroup underwent additional lung function tests, including FEV1 and DLCO (postFEV1 and post-DLCO), at the same facility where the preoperative tests were done. The subgroup's patient characteristics are detailed in Table 1.

The results of the postoperative lung function tests were compared with the predicted values according to the 3 different methods specified.

TABLE 1

VATS Subgroup Patient Characteristics $(n=60)$

\begin{tabular}{lccc}
\hline \multicolumn{1}{c}{ Parameter } & $n(\%)$ & Average & Range \\
\hline Surgery side, right & $31(52 \%)$ & & \\
RUL lobectomy & $19(32 \%)$ & & \\
\hline RML lobectomy & $3(5 \%)$ & & \\
RLL lobectomy & $8(13 \%)$ & & \\
LUL lobectomy & $21(35 \%)$ & & \\
LLL lobectomy & $8(13 \%)$ & & \\
RUL + RML lobectomy & $1(2 \%)$ & & \\
Preoperative FEV1 (\%) & & $74.5 \pm 18$ \\
\hline Preoperative DLCO (\%) & & $68.9 \pm 17$ & $31-127$ \\
\hline Postoperative FEV1 (\%) & & $60.8 \pm 15.7$ & $30-101$ \\
\hline Postoperative DLCO (\%) & & $55.4 \pm 14.3$ & $29-92$ \\
\hline
\end{tabular}

\section{Statistical Methods}

Quantitative variables are presented as mean \pm SD and range. Qualitative variables are described with frequencies and percentages. Statistical analysis was performed with SPSS Statistics, version 25.0 (IBM).

Agreement between quantitative measures was determined by Bland-Altman analysis using a scatterplot for the difference of paired measures and the average of each pair. Limits of agreement were defined as the mean of differences \pm 2 SDs. Paired-sample $t$ testing was used for comparisons between the quantitative measures, and Pearson correlation coefficient testing was used to evaluate the relationship between those measures. The normal distribution of the measures or of the differences between the paired measures was described with histograms and tested with the 1-sample Kolmogrov-Smirnov test. Normality was tested as a preliminary assumption for the Pearson correlation coefficient testing and the paired-sample $t$ test. An $\alpha$-value of less than $5 \%$ was considered significant. A 2-sided significance level is presented, unless otherwise noted.

\section{RESULTS}

\section{Comparison of Relative Uptake Quantified by PS and SPECT/CT}

The relative uptake was calculated for each lung lobe using PS and SPECT/CT quantification in all patients. The mean relative uptake calculated by PS and SPECT/CT and the difference between them is shown in Table 2. A significant difference was found between the quantitative methods in all lung lobes except for the LUL. PS consistently showed higher relative uptake in the RML (mean difference [PS - SPECT/CT], $18.0 \pm 5.0 ; P<$ 0.001 ) and consistently lower results for the RUL and RLL (mean difference [PS - SPECT/CT], $-8.2 \pm 3.8$ and -11.5 \pm 6.1 , respectively; $P<0.001)$. A minor but significant difference was found between the methods in the LLL (mean difference [PS - SPECT/CT], $-2.0 \pm 4.0 ; P<0.001$ ), and no significant difference was found in the LUL (mean difference [PS - SPECT/CT], $-0.4 \pm 4.4 ; P>0.05)$. A difference of more than $10 \%$ in lobar quantification between methods was observed in 26 patients (35\%) in the RUL, 69 patients $(92 \%)$ in the RML, 47 patients $(63 \%)$ in the RLL, 3 patients $(4 \%)$ in the LUL, and 5 patients $(7 \%)$ in the LLL.

Despite the significant difference in relative uptake calculated by PS and SPECT/CT, there was a good correlation between the 2 methods in all lung lobes except the RML (Fig. $2)$. The correlation was stronger in the LUL, LLL, and RUL ( $R=0.67,0.77$, and 0.64 , respectively; $P<0.001)$ and moderate in the RLL $(R=0.49 ; P<0.001)$. A weak correlation, with borderline significance, was found for the RML $(R=0.22$; $P=0.57)$.

\section{Comparison of Predicted Postoperative Lung Function Calculated According to SC, PS, and SPECT/CT and the Actual Postoperative Results}

In the subgroup of 60 patients who underwent VATS lobectomy, $31(52 \%)$ underwent right lobectomy and 29 left lobectomy (48\%). Most lobectomies were of the LUL (21, 35\%) and RUL $(19,32 \%)$, and only 3 patients underwent RML lobectomy (5\%) (Table 1).

The mean ppoFEV1 and ppoDLCO measurements calculated using SC were $58.6 \pm 13.9$ and $54.2 \pm 13.9$, respectively. The mean ppoFEV1 and ppoDLCO were $60.8 \% \pm 14.0 \%$ and $56.5 \% \pm$ $15.3 \%$ when calculated by PS and $60.4 \pm 13.6$ and $55.7 \pm 13.4$ 
TABLE 2

Relative Uptake of Lung Lobes According to Planar and SPECT/CT and Difference Between Methods $(n=75)$

\begin{tabular}{|c|c|c|c|c|c|c|}
\hline \multirow[b]{2}{*}{ Lobe } & \multicolumn{2}{|c|}{ Planar } & \multicolumn{2}{|c|}{ SPECT/CT } & \multirow[b]{2}{*}{ Mean difference } & \multirow[b]{2}{*}{ 2-sided significance } \\
\hline & Mean (\%) & Range (\%) & Mean (\%) & Range (\%) & & \\
\hline RUL & $9( \pm 3)$ & $3-20$ & $18( \pm 5)$ & $7-31$ & $-8.2( \pm 3.8)$ & $<0.001$ \\
\hline $\mathrm{RML}$ & $27( \pm 4)$ & $13-37$ & $10( \pm 4)$ & $3-24$ & $18.0( \pm 5.0)$ & $<0.001$ \\
\hline RLL & $14( \pm 5)$ & $2-27$ & $26( \pm 7)$ & $3-53$ & $-11.5( \pm 6.1)$ & $<0.001$ \\
\hline LUL & $23( \pm 4)$ & $11-34$ & $23( \pm 6)$ & $6-40$ & $-0.4( \pm 4.4)$ & 0.05 (NS) \\
\hline LLL & $26( \pm 6)$ & $6-43$ & $24( \pm 7)$ & $3-40$ & $2.0( \pm 4.0)$ & $<0.001$ \\
\hline
\end{tabular}

NS $=$ No significance

when calculated by SPECT/CT. The actual mean postoperative lung function results were an FEV1 of $60.8 \% \pm 15.7 \%$ and a DLCO of $55.4 \% \pm 14.3 \%$. A significant correlation was found between the predicted FEV1 and DLCO and the postoperative results for all 3 methods (Fig. 3). The ppoFEV1 calculated by SC showed the strongest correlation to the actual postoperative value $(R=0.76 ; P<$ $0.001)$, whereas the SPECT/CT-derived ppoDLCO calculation had the strongest correlation to the postoperative value $(R=0.64 ; P<$ 0.001 ). Bland-Altman plots showed no significant differences between predicted and postoperative values for any of the 3 methods (Fig. 4), with the lowest limits of the 95\% confidence interval being shown by SC for FEV1 ( -18.4 to 22.9) and SPECT/CT for DLCO (-20.6 to 21.5).

A comparison of the values predicted by the different methods and the postoperative measurements showed similar results in patients undergoing either right- or left-sided lobectomy, with correlation coefficients of $0.74,0.70$, and $0.71(P<0.001)$ for SC, $\mathrm{PS}$, and SPECT/CT, respectively, for right-lung lobectomy and $0.80,0.76$, and $0.80(P<0.001)$, respectively, for left-lung lobectomy. Comparison between the methods according to the lobes resected showed similar results in all lobes except the RML, where the ppoFEV1 and ppoDLCO calculated by PS were lower than the results by SC and SPECT/CT and lower than the actual postoperative results (Supplemental Fig. 1; supplemental materials are available at http://jnm.snmjournals.org), though a statistical analysis was not possible since only 3 patients underwent RML lobectomy.

\section{DISCUSSION}

In this prospective study on a homogeneous population of patients with localized non-small cell lung cancer planned for VATS lobectomy, although significant differences were shown between the relative lobar uptake quantified by PS and SPECT/CT in the right lung lobes, no significant differences were found between these methods for predicting postoperative lung function.

These results are consistent with some of the previous studies comparing the role of tomographic techniques such as SPECT/CT to PS (summarized in Table 3) and were validated in this study using strict methodology in patient selection, an advanced SPECT/ CT-derived lung segmentation method, a single facility for lung function testing, and a single surgical method. Previous studies comparing these methods included patients undergoing several types of procedures, including pneumonectomy, lobectomy, and segmentectomy, and several surgical methods, including thoracotomy and VATS $(10,11,17,18)$. Our study population was homogeneous, containing only patients undergoing VATS lobectomy, since in patients undergoing pneumonectomy, PS is sufficient for assessing the relative uptake of the whole lung. VATS lobectomy was the only surgical method used in the current study to avoid differences in the patient recovery process after thoracoscopic procedures and open thoracotomies-differences that can affect postoperative lung function tests.

Several previous studies did not compare the scintigraphyderived results with actual postoperative lung function tests, and some evaluated only FEV1 as a measure of the patient's lung function $(14,15,18,19,21)$. In this study, the predicted results (ppoFEV1 and ppoDLCO) according to the different quantification methods were compared with the postoperative tests, which are considered the gold standard, in a subgroup of 60 patients. We included in our lung function assessment both FEV1 and DLCO, since FEV1 is considered to be the standard reference value but recent studies have shown that DLCO has a better correlation with survival after lobectomy for lung cancer patients $(22,23)$. All patients in the VATS lobectomy subgroup underwent lung function tests before and after surgery, and the tests were conducted during a specified and limited period before and after surgery. Because the results of lung function tests can vary significantly between different facilities, all lung function tests in our study were conducted at a single facility.

The SPECT and CT scans in this study were acquired sequentially, thus avoiding errors of misregistration and enabling attenuation correction of the SPECT data. SPECT/CT quantitative assessment of lung perfusion was performed using the Qlung semiautomatic software, which divides the lung into volumes of interest for each lobe according to the actual lung fissures, thus ensuring that even in patients with significant anatomic changes the segmentation is correct. Other commercial software from different vendors is available for lobar segmentation and will need to be validated separately. Some of the previous studies acquired the SPECT and the CT scans at different time points and coregistered both datasets later $(14,16)$. This method can cause misregistration errors. Other studies used different methods of segmentation of the SPECT/CT data into the lung lobes. For example, the study by Kovacević-Kuśmierek et al. (17) used the CT for finding landmarks, such as the position of the tracheal bifurcation or the fourth rib, for the location of the lung fissures, since the low-dose CT did not visualize the fissures themselves. This method can introduce lung segmentation errors in patients with unusual lung anatomy, especially in patients with gross emphysematous or fibrotic changes. 


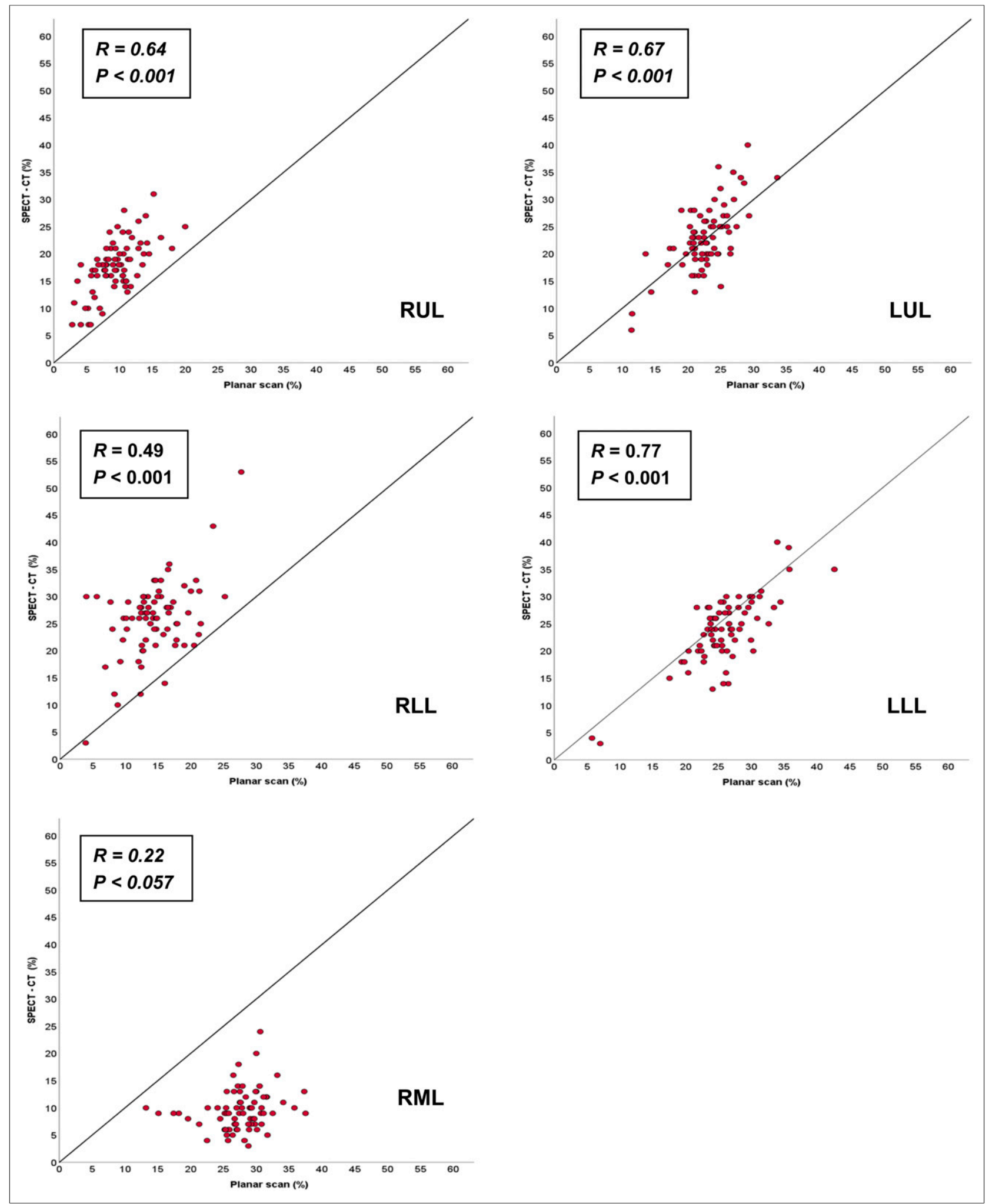

FIGURE 2. Correlation between relative uptake calculated by PS and SPECT/CT for each lobe $(n=75)$.

The comparison between the relative lobar uptake according to PS and SPECT/CT showed significant differences in the quantitation of the right lung lobes but minor differences in the left lung lobes. The difference between the methods was most pronounced in the RML, with a mean difference of $18.0 \pm 5.0$ and a difference of more than $10 \%$ found in $92 \%$ of the patients. This result was probably due to the inability of PS to differentiate between the RML and the remainder of the right lung. Similar results were 


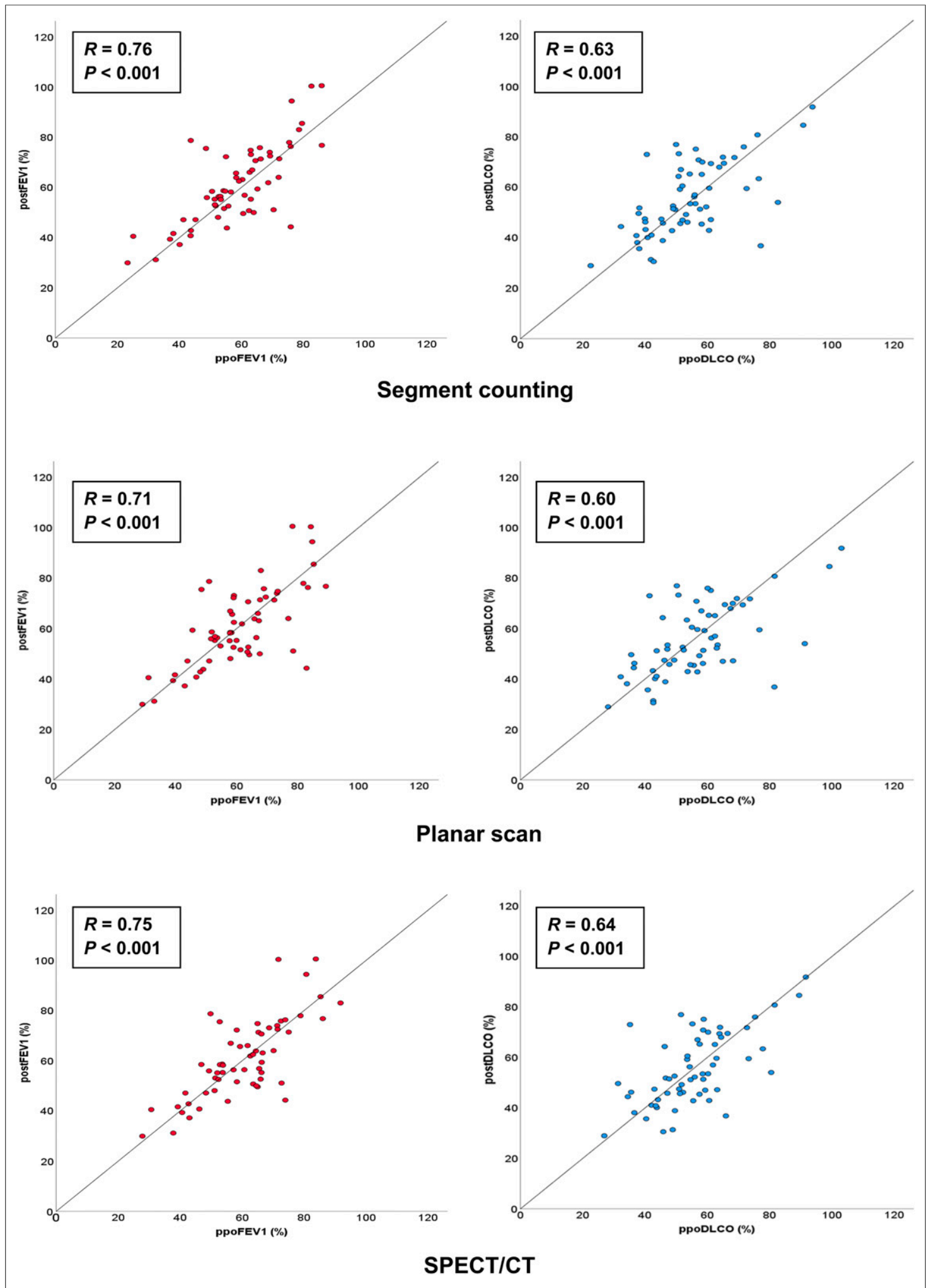

FIGURE 3. Correlation between predicted and postoperative FEV1 and DLCO for 3 methods in VATS lobectomy subgroup $(n=60)$.

shown in studies by Suh et al. (18), Genseke et al. (19), and Provost et al. (24).

Although significant differences were found between the quantification methods, no such differences were shown when the predicted postoperative lung function was calculated according to the different methods in the VATS lobectomy subgroup, including the simple nonimaging SC method. SC had a slightly better performance in predicting the post-FEV1 values and SPECT/CT in 


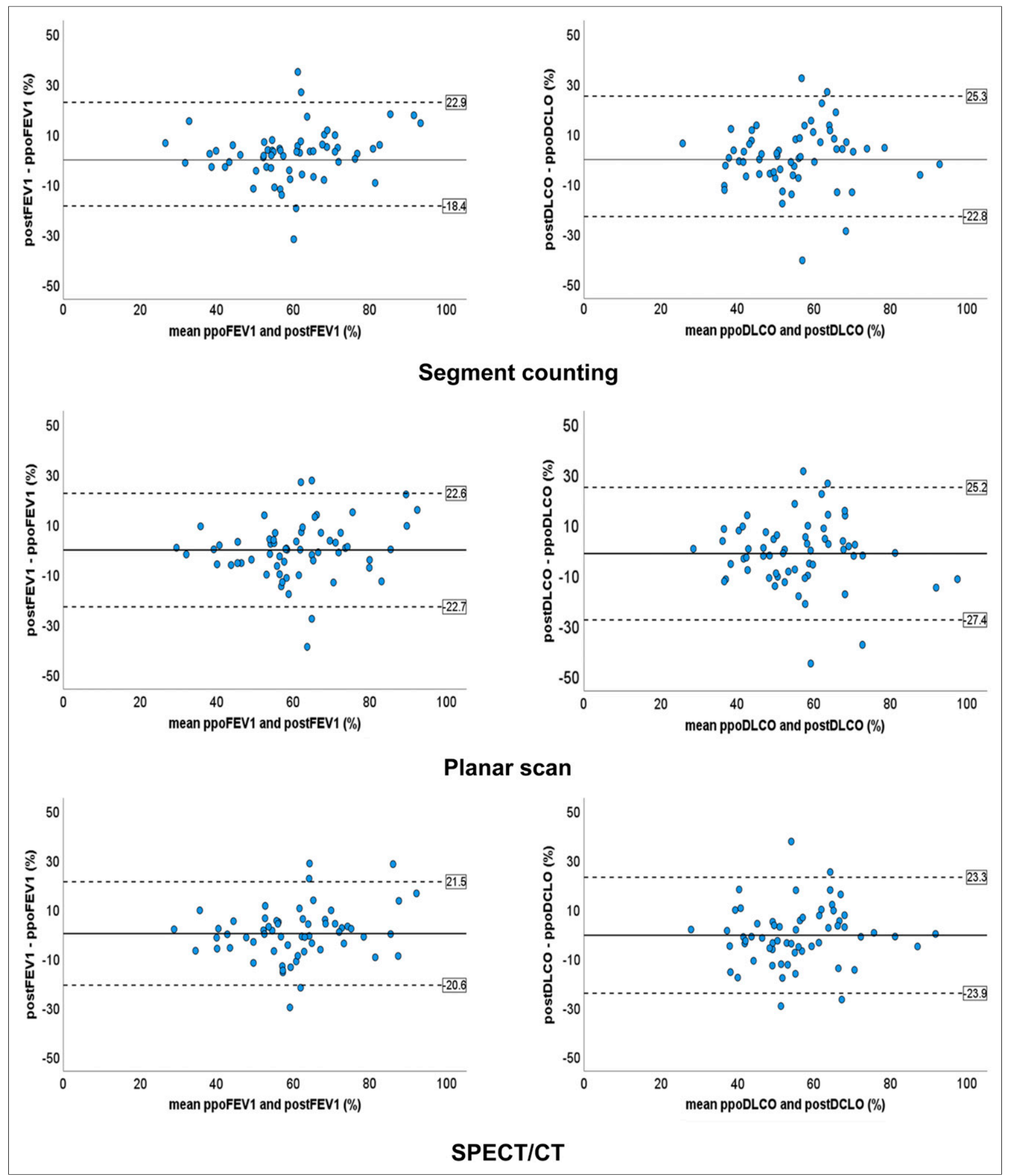

FIGURE 4. Bland-Altman plots comparing predicted and postoperative FEV1 and DLCO for 3 methods in VATS lobectomy subgroup $(n=60)$.

predicting the post-DLCO values, but these were minor differences. We did find a difference, however, in the predicted values for both FEV1 and DLCO between the PS method and the remainder of the methods in RML lobectomy patients, but these could not be statistically proven because of the small number of patients in this group. The study of Suh et al. showed similar results, but only a small portion of patients in the study underwent postoperative lung function tests (9/55 patients) (18).

The major limitation of our study was the relatively small number of patients, especially patients undergoing RML lobectomy. 


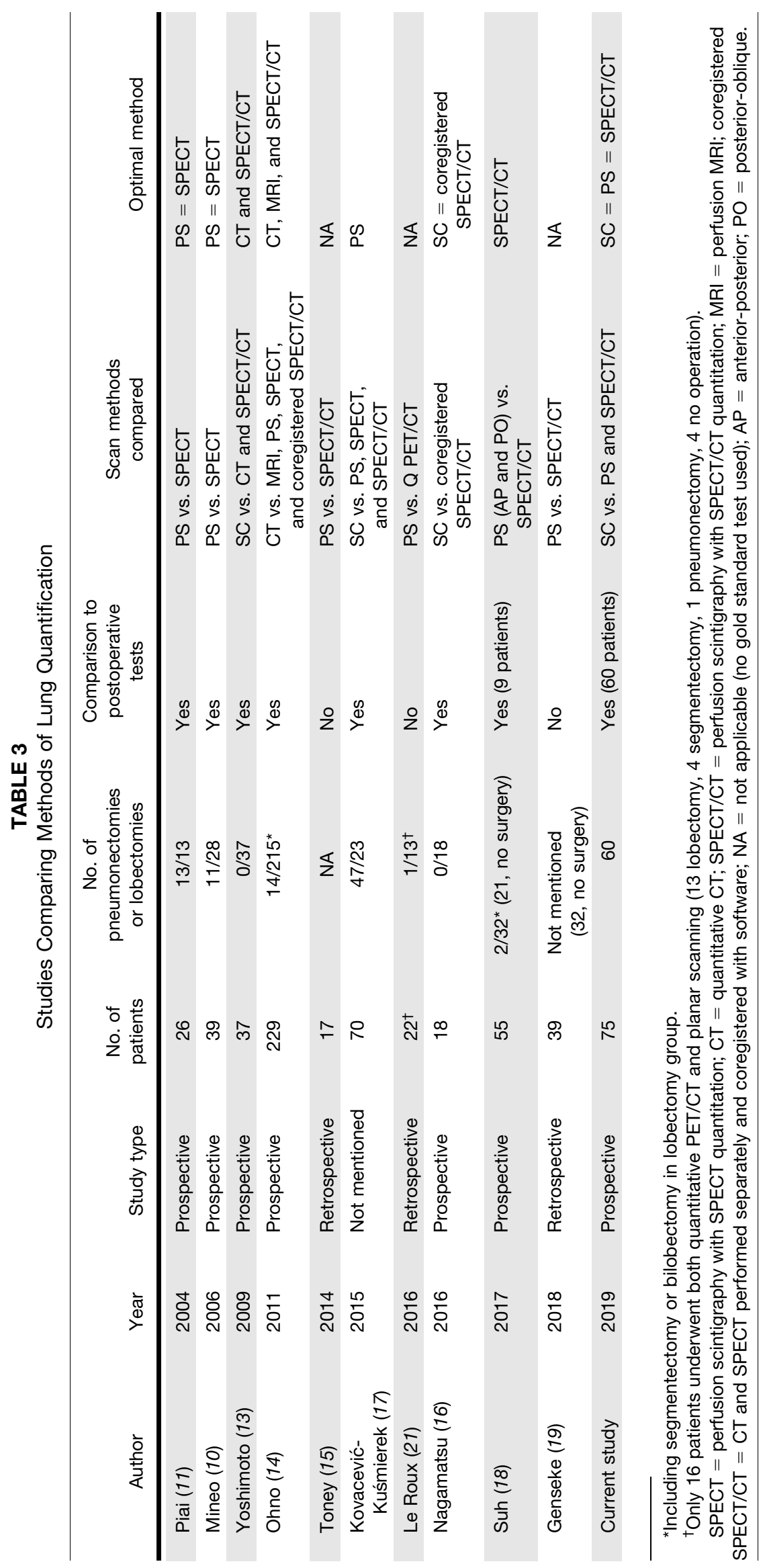

988 The Journal of Nuclear Medicine • Vol. 61 - No. 7 • July 2020 


\section{CONCLUSION}

Lobar quantification significantly differs between PS and SPECT/ $\mathrm{CT}$ in the right lung lobes. SC, PS, and SPECT/CT showed similar performance in prediction of postoperative FEV1 and DLCO. SPECT/CT may have an added value in patients planned for right-lung lobectomy, but further research on a larger patient cohort is needed.

\section{DISCLOSURE}

No potential conflict of interest relevant to this article was reported.

\section{KEY POINTS}

QUESTION: Is SPECT/CT-based quantification of perfusion scan different from PS-based quantification, and which method of predicting postoperative lung function in lobectomy patients is more accurate?

PERTINENT FINDINGS: In this prospective study on 75 patients, SPECT/CT-based quantification of lung function significantly differed from planar-based quantification in the lobes of the right lung. No significant differences were found between predicted lung function calculated with a nonimaging SC method, planar perfusion scanning, SPECT/CT, and the actual postoperative values in a subgroup of 60 patients.

IMPLICATIONS FOR PATIENT CARE: In most lung cancer patients who are candidates for lobectomy, postoperative lung function can be predicted with the simple nonimaging SC method. Lung scintigraphy with SPECT/CT quantification should be considered for right lobectomy over planar-based quantification.

\section{REFERENCES}

1. Torre LA, Siegel RL, Jemal A. Lung cancer statistics. Adv Exp Med Biol. 2016;893:1-19.

2. Maconachie R, Mercer T, Navani N, McVeigh G. Lung cancer: diagnosis and management: summary of updated NICE guidance. Br Med J. 2019;364:11049.

3. Bolliger CT, Koegelenberg CF, Kendal R. Preoperative assessment for lung cancer surgery. Curr Opin Pulm Med. 2005;11:301-306.

4. Little AG, Rusch VW, Bonner JA, et al. Patterns of surgical care of lung cancer patients. Ann Thorac Surg. 2005;80:2051-2056.

5. Brunelli A, Charloux A, Bolliger CT, et al. ERS/ESTS clinical guidelines on fitness for radical therapy in lung cancer patients (surgery and chemo-radiotherapy). Eur Respir J. 2009;34:17-41.

6. Brunelli A, Kim AW, Berger KI, Addrizzo-Harris DJ. Physiologic evaluation of the patient with lung cancer being considered for resectional surgery: diagnosis and management of lung cancer, 3rd ed: American college of chest physicians evidence-based clinical practice guidelines. Chest. 2013;143(5 suppl):e166S-e190S.
7. Zeiher BG, Gross TJ, Kern JA, Lanza LA, Peterson MW. Predicting postoperative pulmonary function in patients undergoing lung resection. Chest. 1995;108: 68-72.

8. Bolliger CT, Gückel C, Engel H, et al. Prediction of functional reserves after lung resection: comparison between quantitative computed tomography, scintigraphy, and anatomy. Respiration. 2002;69:482-489.

9. Corris PA, Ellis DA, Hawkins T, Gibson GJ. Use of radionuclide scanning in the preoperative estimation of pulmonary function after pneumonectomy. Thorax. 1987;42:285-291.

10. Mineo TC, Schillaci O, Pompeo E, Mineo D, Simonetti G. Usefulness of lung perfusion scintigraphy before lung cancer resection in patients with ventilatory obstruction. Ann Thorac Surg. 2006;82:1828-1834.

11. Piai DB, Quagliatto R Jr, Toro I, et al. The use of SPECT in preoperative assessment of patients with lung cancer. Eur Respir J. 2004;24:258-262.

12. Hirose Y, Imaeda T, Doi H, Kokubo M, Sakai S, Hirose H. Lung perfusion SPECT in predicting postoperative pulmonary function in lung cancer. Ann Nucl Med. 1993; 7:123-126.

13. Yoshimoto K, Nomori H, Mori T, et al. Prediction of pulmonary function after lung lobectomy by subsegments counting, computed tomography, single photon emission computed tomography and computed tomography: a comparative study. Eur J Cardiothorac Surg. 2009;35:408-413.

14. Ohno Y, Koyama H, Nogami M, et al. State-of-the-art radiological techniques improve the assessment of postoperative lung function in patients with non-small cell lung cancer. Eur J Radiol. 2011;77:97-104.

15. Toney LK, Wanner M, Miyaoka RS, Alessio AM, Wood DE, Vesselle H. Improved prediction of lobar perfusion contribution using technetium-99m-labeled macroaggregate of albumin single photon emission computed tomography/computed tomography with attenuation correction. J Thorac Cardiovasc Surg. 2014; 148:2345-2352.

16. Nagamatsu Y, Sueyoshi S, Sasahara H, et al. Predicting exercise capacity after lobectomy by single photon emission computed tomography and computed tomography. Gen Thorac Cardiovasc Surg. 2016;64:537-542.

17. Kovacević-Kuśmierek K, Kozak J, Pryt $€$, et al. Perfusion lung scintigraphy for the prediction of postoperative residual pulmonary function in patients with lung cancer. Nucl Med Rev Cent East Eur. 2015;18:70-77.

18. Suh M, Kang YK, Ha S, et al. Comparison of two different segmentation methods on planar lung perfusion scan with reference to quantitative value on SPECT/ CT. Nucl Med Mol Imaging. 2017;51:161-168.

19. Genseke P, Wetz C, Wallbaum T, et al. Lung cancer pre-operative quantification of pulmonary function using hybrid-SPECT/low-dose-CT : a pilot study. Lung Cancer. 2018;118:155-160.

20. Markos J, Mullan BP, Hillman DR, et al. Preoperative assessment as a predictor of mortality and morbidity after lung resection. Am Rev Respir Dis. 1989;139: 902-910.

21. Le Roux PY, Leong TL, Barnett SA, et al. Gallium-68 perfusion positron emission tomography/computed tomography to assess pulmonary function in lung cancer patients undergoing surgery. Cancer Imaging. 2016;16:24.

22. Cerfolio RJ, Bryant AS. Different diffusing capacity of the lung for carbon monoxide as predictors of respiratory morbidity. Ann Thorac Surg. 2009;88: 405-410.

23. Brunelli A, Refai MA, Salati M, Sabbatini A, Morgan-Hughes NJ, Rocco G. Carbon monoxide lung diffusion capacity improves risk stratification in patients without airflow limitation: evidence for systematic measurement before lung resection. Eur J Cardiothorac Surg. 2006;29:567-570.

24. Provost K, Leblond A, Gauthier-Lemire A, Filion E, Bahig H, Lord M. Reproducibility of lobar perfusion and ventilation quantification using SPECT/CT segmentation software in lung cancer patients. J Nucl Med Technol. 2017;45: $185-192$. 